\title{
On vector matrix game and symmetric dual vector optimization problem
}

\author{
Jeong Min Hong ${ }^{1}$, Moon Hee Kim² and Gue Myung Lee ${ }^{1 *}$
}

${ }^{\text {*Correspondence: }}$ gmlee@pknu.ac.kr

1 Department of Applied

Mathematics, Pukyong National

University, Busan, 608-737, Korea

Full list of author information is

available at the end of the article

\begin{abstract}
A vector matrix game with more than two skew symmetric matrices, which is an extension of the matrix game, is defined and the symmetric dual problem for a nonlinear vector optimization problem is considered. Using the Kakutani fixed point theorem, we prove an existence theorem for a vector matrix game. We establish equivalent relations between the symmetric dual problem and its related vector matrix game. Moreover, we give an example illustrating the equivalent relations.
\end{abstract}

\section{Introduction}

A matrix game is defined by $B$ of a real $m \times n$ matrix together with the Cartesian product $S_{n} \times S_{m}$ of all $n$-dimensional probability vectors $S_{n}$ and all $m$-dimensional probability vectors $S_{m}$; that is, $S_{n}:=\left\{x=\left(x_{1}, \ldots, x_{n}\right)^{T} \in \mathbb{R}^{n}: x_{i} \geqq 0, \sum_{i=1}^{n} x_{i}=1\right\}$, where the symbol ${ }^{T}$ denotes the transpose. A point $(\bar{x}, \bar{y}) \in S_{n} \times S_{n}$ is called an equilibrium point of a matrix game $B$ if $x^{T} B \bar{y} \leqq \bar{x}^{T} B \bar{y} \leqq \bar{x}^{T} B y$ for all $x, y \in S_{n}$ and $\bar{x} B \bar{y}=v$, where $v$ is value of the game. If $n=m$ and $B$ is skew symmetric, then we can check that $(\bar{x}, \bar{y}) \in S_{n} \times S_{n}$ is an equilibrium point of the game $B$ if and only if $B \bar{x} \leqq 0$ and $B \bar{y} \leqq 0$. When $B$ is an $n \times n$ skew symmetric matrix, $\bar{x} \in S_{n}$ is called a solution of the matrix game $B$ if $B \bar{x} \leqq 0$ [1].

Consider the linear programming problem (LP) and its dual (LD) as follows:

(LP) Minimize $c^{T} x$ subject to $A x \geqq b, x \geqq 0$,

(LD) Maximize $b^{T} y \quad$ subject to $A^{T} y \leqq c, y \geqq 0$,

where $c \in \mathbb{R}^{n}, x \in \mathbb{R}^{n}, b \in \mathbb{R}^{m}, y \in \mathbb{R}^{m}, A=\left[a_{i j}\right]$ is an $m \times n$ real matrix.

Now consider the matrix game associated with the following $(n+m+1) \times(n+m+1)$ skew symmetric matrix $B$ :

$$
B=\left[\begin{array}{ccc}
0 & A^{T} & -c \\
-A & 0 & b \\
c^{T} & -b^{T} & 0
\end{array}\right] .
$$

Dantzig [1] gave the complete equivalence between the linear programming duality and the matrix game $B$. Many authors [2-5] have extended the equivalence results of Dantzig [1] to several kinds of scalar optimization problems. Very recently, Hong and Kim [6] defined a vector matrix game and generalized the equivalence results of Dantzig [1] to a vector optimization problem by using the vector matrix game.

Recently, Kim and Noh [4] established equivalent relations between a certain matrix game and symmetric dual problems. Symmetric duality in nonlinear programming, in

(c) 2012 Hong et al.; licensee Springer. This is an Open Access article distributed under the terms of the Creative Commons Attribution License (http://creativecommons.org/licenses/by/2.0), which permits unrestricted use, distribution, and reproduction in any medium, provided the original work is properly cited. 
which the dual of the dual is the primal, was first introduced by Dorn [7]. Dantzig, Eisenberg and Cottle [8] formulated a pair of symmetric dual nonlinear problems and established duality results for convex and concave functions with non-negative orthant as the cone. Mond and Weir [9] presented two pairs of symmetric dual vector optimization problems and obtained symmetric duality results concerning pseudoconvex and pseudoconcave functions.

In this paper, a vector matrix game with more than two skew symmetric matrices, which is an extension of the matrix game, is defined and a nonlinear vector optimization problem is considered. We formulate a symmetric dual problem for the nonlinear vector optimization problem and establish equivalent relations between the symmetric dual problem and the corresponding vector matrix game. Moreover, we give a numerical example for showing such equivalent relations.

\section{Vector matrix game and existence theorem}

Throughout this paper, we will denote the relative interior of $S_{p}$ by $\stackrel{o}{S}_{p}$, and we will use the following conventions for vectors in the Euclidean space $\mathbb{R}^{n}$ for vectors $x:=\left(x_{1}, \ldots, x_{n}\right)$ and $y:=\left(y_{1}, \ldots, y_{n}\right):$

$$
\begin{aligned}
& x \leqq y \text { if and only if } x_{i} \leqq y_{i}, \quad i=1, \ldots, n ; \\
& x<y \text { if and only if } x_{i}<y_{i}, \quad i=1, \ldots, n ; \\
& x \leq y \text { if and only if } x_{i} \leqq y_{i}, \text { and } x \neq y ; \text { and } \\
& x \not y \text { is the negation of } x \leq y .
\end{aligned}
$$

Consider the nonlinear programming problem (VOP):

$$
\begin{gathered}
\text { (VOP) Minimize } f(x):=\left(f_{1}(x), \ldots, f_{p}(x)\right) \\
\text { subject to } x \in X,
\end{gathered}
$$

where $X=\left\{x \in \mathbb{R}^{n}: g(x) \geqq b, x \geqq 0\right\}, f: \mathbb{R}^{n} \rightarrow \mathbb{R}^{p}, g: \mathbb{R}^{n} \rightarrow \mathbb{R}^{m}$ are continuously differentiable. The gradient $\nabla f(x)$ is an $n \times p$ matrix, and $\nabla g(x)$ is an $n \times m$ matrix.

Definition 2.1 [10] A point $\bar{x} \in X$ is said to be an efficient solution for (VOP) if there exists no other feasible point $x \in X$ such that $\left(f_{1}(x), \ldots, f_{p}(x)\right) \leq\left(f_{1}(\bar{x}), \ldots, f_{p}(\bar{x})\right)$.

Now, we define solutions for a vector matrix game as follows.

Definition 2.2 [6] Let $B_{i}, i=1, \ldots, p$, be real $n \times n$ skew-symmetric matrices. A point $\bar{x} \in S_{n}$ is said to be a vector solution of the vector matrix game $B_{i}, i=1, \ldots, p$ if $\left(\bar{x}^{T} B_{1} x, \ldots, \bar{x}^{T} B_{p} x\right) \not \leq\left(\bar{x}^{T} B_{1} \bar{x}, \ldots, \bar{x}^{T} B_{p} \bar{x}\right) \not\left(x^{T} B_{1} \bar{x}, \ldots, x^{T} B_{p} \bar{x}\right)$ for any $x \in S_{n}$.

We proved the characterization of a vector solution of the vector matrix game in [6].

Lemma 2.1 [6] Let $B_{i}, i=1, \ldots, p$, be an $n \times n$ skew symmetric matrix. Then $\bar{y} \in S_{n}$ is a vector solution of the vector matrix game $B_{i}, i=1, \ldots, p$, if and only if there exists $\xi \in \stackrel{o}{S}_{p}$ such that $\left(\sum_{i=1}^{p} \xi_{i} B_{i}\right) \bar{y} \leqq 0$. 
Remark 2.1 Let $B_{i}, i=1, \ldots, p$, be an $n \times n$ skew symmetric matrix. From Lemma 2.1, we can obtain the following remark saying that the vector matrix game can be solved by fixed point problems; $\bar{y} \in S_{n}$ is a vector solution of the vector matrix game $B_{i}, i=1, \ldots, p$, if and only if there exists $\xi \in \stackrel{o}{S}_{p}$ such that $\bar{y} \in F_{\xi}(\bar{y})$, where $F_{\xi}(x)=\left\{y \in S_{n} \mid y \in x-\left(\sum_{i=1}^{p} \xi_{i} B_{i}\right) x-\right.$ $\left.\mathbb{R}_{+}^{n}\right\}$.

Noticing Remark 2.1, we can obtain an existence theorem for the vector matrix game.

Theorem 2.1 Let $B_{i}, i=1, \ldots, p$, be an $n \times n$ skew symmetric matrix. Then there exists a vector solution of the vector matrix game $B_{i}, i=1, \ldots, p$.

Proof Let $\xi \in \stackrel{o}{S}$. Define a multifunction $F_{\xi}: S_{n} \rightarrow S_{n}$ by, for any $x \in S_{n}$,

$$
F_{\xi}(x)=\left\{y \in S_{n} \mid y \in x-\left(\sum_{i=1}^{p} \xi_{i} B_{i}\right) x-\mathbb{R}_{+}^{n}\right\} .
$$

Then the multifunction $F_{\xi}$ is closed and hence upper semi-continuous, and so it follows from the well-known Kakutani fixed point theorem [11] that the multifunction $F_{\xi}$ has a fixed point. So, by Remark 2.1, there exists a vector solution of the vector matrix game $B_{i}$, $i=1, \ldots, p$.

\section{Equivalence relations}

Now, we consider the nonlinear symmetric programming problem (SP) together with its dual (SD) as follows:

$$
\begin{aligned}
\text { Minimize } & \left(f_{1}(x, y)-y^{T} \nabla_{y}\left(\lambda^{T} f\right)(x, y), \ldots, f_{p}(x, y)-y^{T} \nabla_{y}\left(\lambda^{T} f\right)(x, y)\right) \\
\text { subject to } & -\nabla_{y}\left(\lambda^{T} f\right)(x, y) \geqq 0, \\
& x \geqq 0, \quad \lambda>0, \\
\text { Maximize } \quad & \left(f_{1}(u, v)-u^{T} \nabla_{u}\left(\lambda^{T} f\right)(u, v), \ldots, f_{p}(u, v)-u^{T} \nabla_{u}\left(\lambda^{T} f\right)(u, v)\right) \\
\text { subject to } & -\nabla_{u}\left(\lambda^{T} f\right)(u, v) \leqq 0, \\
& v \geqq 0, \quad \lambda>0,
\end{aligned}
$$

where $f:=\left(f_{1}, \ldots, f_{p}\right): \mathbb{R}^{n} \times \mathbb{R}^{m} \rightarrow \mathbb{R}^{p}$ are continuously differentiable.

Consider the vector matrix game defined by the following $(n+m+1) \times(n+m+1)$ skew symmetric matrix $B_{i}(x, y), i=1, \ldots, p$, related to (SP) and (SD):

$$
B_{i}(x, y)=\left[\begin{array}{ccc}
0 & -x \nabla_{y} f_{i}(x, y)^{T} & -\nabla_{x} f_{i}(x, y) \\
\nabla_{y} f_{i}(x, y) x^{T} & 0 & \nabla_{y} f_{i}(x, y) \\
\nabla_{x} f_{i}(x, y)^{T} & -\nabla_{y} f_{i}(x, y)^{T} & 0
\end{array}\right] .
$$

Now, we give equivalent relations between (SD) and the vector matrix game $B_{i}(x, y)$, $i=1, \ldots, p$.

Theorem 3.1 Let $(\bar{x}, \bar{y}, \bar{\xi})$ be feasible for (SP) and (SD), with $\bar{y}^{T} \nabla_{y}\left(\bar{\xi}^{T} f\right)(\bar{x}, \bar{y})=\bar{x}^{T} \nabla_{x}\left(\bar{\xi}^{T} f\right) \times$ $(\bar{x}, \bar{y})=0$. Let $z^{*}=1 /\left(1+\sum_{i} \bar{x}_{i}+\sum_{j} \bar{y}_{j}\right), x^{*}=z^{*} \bar{x}$ and $y^{*}=z^{*} \bar{y}$. Then $\left(x^{*}, y^{*}, z^{*}\right)$ is a vector solution of the vector matrix game $B_{i}(\bar{x}, \bar{y}), i=1, \ldots, p$. 
Proof Let $(\bar{x}, \bar{y}, \bar{\xi})$ be feasible for (SP) and (SD). Then the following holds:

$$
\begin{aligned}
& -\nabla_{y}\left(\bar{\xi}^{T} f\right)(\bar{x}, \bar{y}) \geqq 0, \\
& -\nabla_{x}\left(\bar{\xi}^{T} f\right)(\bar{x}, \bar{y}) \leqq 0, \\
& \bar{y}^{T} \nabla_{y}\left(\bar{\xi}^{T} f\right)(\bar{x}, \bar{y})=\bar{x}^{T} \nabla_{x}\left(\bar{\xi}^{T} f\right)(\bar{x}, \bar{y})=0, \\
& \bar{x} \geqq 0, \quad \bar{y} \geqq 0, \quad \bar{\xi} \in \stackrel{o}{S}_{p} .
\end{aligned}
$$

Multiplying (3.3) by $\bar{x} \geqq 0$ gives $-\bar{x} \nabla_{y}\left(\bar{\xi}^{T} f\right)(\bar{x}, \bar{y})^{T} \bar{y}=0$ and from (3.2),

$$
-\bar{x} \nabla_{y}\left(\bar{\xi}^{T} f\right)(\bar{x}, \bar{y})^{T} \bar{y}-\nabla_{x}\left(\bar{\xi}^{T} f\right)(\bar{x}, \bar{y}) \leqq 0 .
$$

Multiplying (3.1) by $\bar{x}^{T} \bar{x} \geqq 0, \nabla_{y}\left(\bar{\xi}^{T} f\right)(\bar{x}, \bar{y}) \bar{x}^{T} \bar{x} \leqq 0$. It implies that since $\nabla_{y}\left(\bar{\xi}^{T} f\right)(\bar{x}, \bar{y}) \leqq 0$,

$$
\nabla_{y}\left(\bar{\xi}^{T} f\right)(\bar{x}, \bar{y}) \bar{x}^{T} \bar{x}+\nabla_{y}\left(\bar{\xi}^{T} f\right)(\bar{x}, \bar{y}) \leqq 0 .
$$

From (3.3) we have

$$
\nabla_{x}\left(\bar{\xi}^{T} f\right)(\bar{x}, \bar{y})^{T} \bar{x}-\nabla_{y}\left(\bar{\xi}^{T} f\right)(\bar{x}, \bar{y})^{T} \bar{y}=0 .
$$

But $z^{*}>0$ by (3.4), from (3.5), (3.6) and (3.7), we get

$$
\begin{aligned}
& -\bar{x} \nabla_{y}\left(\bar{\xi}^{T} f\right)(\bar{x}, \bar{y})^{T} y^{*}-\nabla_{x}\left(\bar{\xi}^{T} f\right)(\bar{x}, \bar{y}) z^{*} \leqq 0, \\
& \nabla_{y}\left(\bar{\xi}^{T} f\right)(\bar{x}, \bar{y}) \bar{x}^{T} x^{*}+\nabla_{y}\left(\bar{\xi}^{T} f\right)(\bar{x}, \bar{y}) z^{*} \leqq 0, \\
& \nabla_{x}\left(\bar{\xi}^{T} f\right)(\bar{x}, \bar{y})^{T} x^{*}-\nabla_{y}\left(\bar{\xi}^{T} f\right)(\bar{x}, \bar{y})^{T} y^{*}=0, \\
& x^{*} \geqq 0, \quad y^{*} \geqq 0, \quad z^{*}>0 .
\end{aligned}
$$

From (3.8), (3.9) and (3.10), we have the following inequality:

$$
\left(\sum_{i=1}^{p} \bar{\xi}_{i} B_{i}(\bar{x}, \bar{y})\right)\left(\begin{array}{l}
x^{*} \\
y^{*} \\
z^{*}
\end{array}\right) \leqq 0 .
$$

By Lemma $2.1,\left(x^{\prime \prime}, y^{\prime \prime}, z^{*}\right)$ is a vector solution of the vector matrix game $B_{i}(\bar{x}, \bar{y}), i=1, \ldots, p$.

Theorem 3.2 Let $\left(x^{*}, y^{*}, z^{*}\right)$ with $z^{*}>0$ be a vector solution of the vector matrix game $B_{i}(\bar{x}, \bar{y}), i=1, \ldots, p$, where $\bar{x}=x^{*} / z^{*}$ and $\bar{y}=y^{*} / z^{*}$. Then there exists $\bar{\xi} \in S_{\text {S }}^{o}$ such that $(\bar{x}, \bar{y}, \bar{\xi})$ is feasible for (SP) and (SD), and $\bar{y}^{T} \nabla_{y}\left(\bar{\xi}^{T} f\right)(\bar{x}, \bar{y})=\bar{x}^{T} \nabla_{x}\left(\bar{\xi}^{T} f\right)(\bar{x}, \bar{y})=0$. Moreover, if $f_{i}(\cdot, y)$, $i=1, \ldots, p$, are convex for fixed $y$ and $f_{i}(x, \cdot), i=1, \ldots, p$, are concave for fixed $x$, then $(\bar{x}, \bar{y})$ is efficient for (SP) with fixed $\bar{\xi}$ and $(\bar{x}, \bar{y})$ is efficient for (SD) with fixed $\bar{\xi}$.

Proof Let $\left(x^{*}, y^{*}, z^{*}\right)$ with $z^{*}>0$ be a vector solution of the vector matrix game $B_{i}(\bar{x}, \bar{y})$, $i=1, \ldots, p$. Then by Lemma 2.1 , there exists $\bar{\xi} \in \stackrel{o}{S}_{p}$ such that

$$
\left(\sum_{i=1}^{p} \bar{\xi}_{i} B_{i}(\bar{x}, \bar{y})\right)\left(\begin{array}{l}
x^{\prime \prime} \\
y^{*} \\
z^{*}
\end{array}\right) \leqq 0 .
$$


Thus, we get

$$
\begin{aligned}
& -\bar{x} \nabla_{y}\left(\bar{\xi}^{T} f\right)(\bar{x}, \bar{y})^{T} y^{*}-\nabla_{x}\left(\bar{\xi}^{T} f\right)(\bar{x}, \bar{y}) z^{*} \leqq 0, \\
& \nabla_{y}\left(\bar{\xi}^{T} f\right)(\bar{x}, \bar{y}) \bar{x}^{T} x^{*}+\nabla_{y}\left(\bar{\xi}^{T} f\right)(\bar{x}, \bar{y}) z^{*} \leqq 0, \\
& \nabla_{x}\left(\bar{\xi}^{T} f\right)(\bar{x}, \bar{y})^{T} x^{*}-\nabla_{y}\left(\bar{\xi}^{T} f\right)(\bar{x}, \bar{y})^{T} y^{*} \leqq 0, \\
& x^{*} \geqq 0, \quad y^{*} \geqq 0, \quad z^{*}>0 .
\end{aligned}
$$

Dividing (3.11), (3.12) and (3.13) by $z^{*}>0$, we have

$$
\begin{aligned}
& -\bar{x} \nabla_{y}\left(\bar{\xi}^{T} f\right)(\bar{x}, \bar{y})^{T} \bar{y}-\nabla_{x}\left(\bar{\xi}^{T} f\right)(\bar{x}, \bar{y}) \leqq 0, \\
& \nabla_{y}\left(\bar{\xi}^{T} f\right)(\bar{x}, \bar{y}) \bar{x}^{T} \bar{x}+\nabla_{y}\left(\bar{\xi}^{T} f\right)(\bar{x}, \bar{y}) \leqq 0, \\
& \nabla_{x}\left(\bar{\xi}^{T} f\right)(\bar{x}, \bar{y})^{T} \bar{x}-\nabla_{y}\left(\bar{\xi}^{T} f\right)(\bar{x}, \bar{y})^{T} \bar{y} \leqq 0 .
\end{aligned}
$$

From (3.14),

$$
\bar{x} \geqq 0, \quad \bar{y} \geqq 0 .
$$

By (3.16), $\nabla_{y}\left(\bar{\xi}^{T} f\right)(\bar{x}, \bar{y})\left(\bar{x}^{T} \bar{x}+1\right) \leqq 0$. It implies that since $\bar{x}^{T} \bar{x}+1>0$,

$$
-\nabla_{y}\left(\bar{\xi}^{T} f\right)(\bar{x}, \bar{y}) \geqq 0
$$

From (3.15), $-\bar{x} \nabla_{y}\left(\bar{\xi}^{T} f\right)(\bar{x}, \bar{y})^{T} \bar{y} \leqq \nabla_{x}\left(\bar{\xi}^{T} f\right)(\bar{x}, \bar{y})$. Using (3.18) and (3.19), we obtain $0 \leqq$ $-\bar{x} \nabla_{y}\left(\bar{\xi}^{T} f\right)(\bar{x}, \bar{y})^{T} \bar{y} \leqq \nabla_{x}\left(\bar{\xi}^{T} f\right)(\bar{x}, \bar{y})$. It implies that $-\nabla_{x}\left(\bar{\xi}^{T} f\right)(\bar{x}, \bar{y}) \leqq 0$. From (3.17), $\bar{x}^{T} \nabla_{x}\left(\bar{\xi}^{T} f\right)(\bar{x}, \bar{y}) \leqq \bar{y}^{T} \nabla_{y}\left(\bar{\xi}^{T} f\right)(\bar{x}, \bar{y})$. But since $\bar{x} \geqq 0$ and $\nabla_{x}\left(\bar{\xi}^{T} f\right)(\bar{x}, \bar{y}) \geqq 0, \bar{x}^{T} \nabla_{x}\left(\bar{\xi}^{T} f\right)(\bar{x}, \bar{y}) \geqq$ 0 and since $\bar{y} \geqq 0$ and $\nabla_{y}\left(\bar{\xi}^{T} f\right)(\bar{x}, \bar{y}) \leqq 0, \bar{y}^{T} \nabla_{y}\left(\bar{\xi}^{T} f\right)(\bar{x}, \bar{y}) \leqq 0$. Then we have

$$
0 \leqq \bar{x}^{T} \nabla_{x}\left(\bar{\xi}^{T} f\right)(\bar{x}, \bar{y}) \leqq \bar{y}^{T} \nabla_{y}\left(\bar{\xi}^{T} f\right)(\bar{x}, \bar{y}) \leqq 0
$$

Hence, $\bar{x}^{T} \nabla_{x}\left(\bar{\xi}^{T} f\right)(\bar{x}, \bar{y})=\bar{y}^{T} \nabla_{y}\left(\bar{\xi}^{T} f\right)(\bar{x}, \bar{y})$. Thus, $(\bar{x}, \bar{y}, \bar{\xi})$ is feasible for (SP) and (SD) with $f_{i}(\bar{x}, \bar{y})-\bar{y}^{T} \nabla_{y}\left(\bar{\xi}^{T} f\right)(\bar{x}, \bar{y})=f_{i}(\bar{x}, \bar{y})-\bar{x}^{T} \nabla_{x}\left(\bar{\xi}^{T} f\right)(\bar{x}, \bar{y}), i=1, \ldots, p$. Since $(\bar{x}, \bar{y}, \bar{\xi})$ is feasible for (SD), by weak duality in [9], $\left(f_{1}(x, y)-y^{T} \nabla_{y}\left(\xi^{T} f\right)(x, y), \ldots, f_{p}(x, y)-y^{T} \nabla_{y}\left(\xi^{T} f\right)(x, y)\right) \not$ $\left(f_{1}(\bar{x}, \bar{y})-\bar{y}^{T} \nabla_{y}\left(\bar{\xi}^{T} f\right)(\bar{x}, \bar{y}), \ldots, f_{p}(\bar{x}, \bar{y})-\bar{y}^{T} \nabla_{y}\left(\bar{\xi}^{T} f\right)(\bar{x}, \bar{y})\right)$ and $\left(f_{1}(\bar{x}, \bar{y})-\bar{x}^{T} \nabla_{x}\left(\bar{\xi}^{T} f\right)(\bar{x}, \bar{y}), \ldots, f_{p}(\bar{x}, \bar{y})-\right.$ $\left.\bar{x}^{T} \nabla_{x}\left(\bar{\xi}^{T} f\right)(\bar{x}, \bar{y})\right) \not \leq\left(f_{1}(u, v)-u^{T} \nabla_{u}\left(\xi^{T} f\right)(u, v), \ldots, f_{p}(u, v)-u^{T} \nabla_{u}\left(\xi^{T} f\right)(u, v)\right)$ for any feasible $(u, v, \xi)$ of (SP) and (SD). Therefore, $(\bar{x}, \bar{y})$ is efficient for (SP) with fixed $\bar{\xi}$ and $(\bar{x}, \bar{y})$ is efficient for (SD) with fixed $\bar{\xi}$.

Now, we give an example illustrating Theorems 3.1 and 3.2.

Example 3.1 Let $f_{1}(x, y)=x^{2}-y^{2}$ and $f_{2}(x, y)=y-x$. Consider the following vector optimization problem (SP) together with its dual (SD) as follows:

$$
\text { Minimize }\left(x^{2}-y^{2}+2 \lambda_{1} y^{2}-\lambda_{2} y, y-x+2 \lambda_{1} y^{2}-\lambda_{2} y\right)
$$$$
\text { subject to } 2 \lambda_{1} y-\lambda_{2} \geqq 0 \text {, }
$$

$$
x \geqq 0, \quad \lambda=\left(\lambda_{1}, \lambda_{2}\right) \in \stackrel{o}{S}_{2},
$$


(SD) Maximize $\left(u^{2}-v^{2}-2 \lambda_{1} u^{2}+\lambda_{2} u, v-u-2 \lambda_{1} u^{2}+\lambda_{2} u\right)$

subject to $2 \lambda_{1} u-\lambda_{2} \geqq 0$,

$$
v \geqq 0, \quad \lambda=\left(\lambda_{1}, \lambda_{2}\right) \in \stackrel{o}{S_{2}} .
$$

Now, we determine the set of all vector solutions of the vector matrix game $B_{i}(x, y), i=1,2$.

Let

$$
B_{i}(x, y)=\left(\begin{array}{ccc}
0 & -x \nabla_{y} f_{i}(x, y)^{T} & -\nabla_{x} f_{i}(x, y) \\
-\nabla_{y} f_{i}(x, y) x^{T} & 0 & \nabla_{y} f_{i}(x, y) \\
\nabla_{x} f_{i}(x, y)^{T} & -\nabla_{y} f_{i}(x, y)^{T} & 0
\end{array}\right) .
$$

Then

$$
B_{1}(x, y)=\left(\begin{array}{ccc}
0 & 2 x y & -2 x \\
-2 x y & 0 & -2 y \\
2 x & 2 y & 0
\end{array}\right) \text { and } B_{2}(x, y)=\left(\begin{array}{ccc}
0 & -x & 1 \\
x & 0 & 1 \\
-1 & -1 & 0
\end{array}\right)
$$

Let $(x, y) \in \mathbb{R}^{2}$ and $\left(x^{*}, y^{*}, z^{*}\right) \in S_{3}$ be a vector solution of the vector matrix game $B_{i}(x, y)$, $i=1,2$, if and only if there exist $\xi_{1}>0, \xi_{2}>0, \xi_{1}+\xi_{2}=1$ such that

$$
\left(\xi_{1}\left(\begin{array}{ccc}
0 & 2 x y & -2 x \\
-2 x y & 0 & -2 y \\
2 x & 2 y & 0
\end{array}\right)+\xi_{2}\left(\begin{array}{ccc}
0 & -x & 1 \\
x & 0 & 1 \\
-1 & -1 & 0
\end{array}\right)\right)\left(\begin{array}{l}
x^{*} \\
y^{* \prime} \\
z^{*}
\end{array}\right) \leqq\left(\begin{array}{l}
0 \\
0 \\
0
\end{array}\right) .
$$

$\Longleftrightarrow$ there exist $\xi_{1}>0, \xi_{2}>0, \xi_{1}+\xi_{2}=1$ such that

$$
\left(\begin{array}{c}
x\left(2 y \xi_{1}-\xi_{2}\right) y^{*}-\left(2 x \xi_{1}-\xi_{2}\right) z^{*} \\
-x\left(2 y \xi_{1}-\xi_{2}\right) x^{\prime \prime}-\left(2 y \xi_{1}-\xi_{2}\right) z^{\prime \prime} \\
\left(2 x \xi_{1}-\xi_{2}\right) x^{\prime \prime}+\left(2 y \xi_{1}-\xi_{2}\right) y^{\prime \prime}
\end{array}\right) \leqq\left(\begin{array}{l}
0 \\
0 \\
0
\end{array}\right)
$$

Thus, we determine the set of all the vector solutions of the vector matrix game $B_{i}(x, y)$, $i=1,2$.

(I) the case that $x>0$ :

(a) $2 x \xi_{1}-\xi_{2}>0,2 y \xi_{1}-\xi_{2}>0:\left(x^{\prime \prime}, y^{\prime \prime}, z^{\prime \prime}\right)=(0,0,1)$.

(b) $2 x \xi_{1}-\xi_{2}>0,2 y \xi_{1}-\xi_{2}=0:\left(x^{\prime \prime}, y^{\prime \prime}, z^{\prime \prime}\right):\{(0, \alpha, 1-\alpha) \mid 0 \leqq \alpha \leqq 1\}$.

(c) $2 x \xi_{1}-\xi_{2}>0,2 y \xi_{1}-\xi_{2}<0:\left(x^{*}, y^{*}, z^{*}\right)=(0,1,0)$.

(d) $2 x \xi_{1}-\xi_{2}=0,2 y \xi_{1}-\xi_{2}>0:\left(x^{*}, y^{*}, z^{*}\right):\{(\alpha, 0,1-\alpha) \mid 0 \leqq \alpha \leqq 1\}$.

(e) $2 x \xi_{1}-\xi_{2}=0,2 y \xi_{1}-\xi_{2}=0$ : $\left(x^{*}, y^{*}, z^{*}\right):\left\{\left(x_{1}, x_{2}, x_{3}\right) \mid x_{1} \geqq 0, x_{2} \geqq 0, x_{3} \geqq 0, x_{1}+x_{2}+x_{3}=1\right\}$.

(f) $2 x \xi_{1}-\xi_{2}=0,2 y \xi_{1}-\xi_{2}<0:\left(x^{\prime \prime}, y^{\prime \prime}, z^{\prime \prime}\right)=(0,1,0)$.

(g) $2 x \xi_{1}-\xi_{2}<0,2 y \xi_{1}-\xi_{2}>0:\left(x^{\prime \prime}, y^{\prime \prime}, z^{\prime \prime}\right)=(1,0,0)$.

(h) $2 x \xi_{1}-\xi_{2}<0,2 y \xi_{1}-\xi_{2}=0:\left(x^{\prime \prime}, y^{\prime \prime}, z^{\prime \prime}\right):\{(\alpha, 1-\alpha, 0) \mid 0 \leqq \alpha \leqq 1\}$.

(i) $2 x \xi_{1}-\xi_{2}<0,2 y \xi_{1}-\xi_{2}<0:\left(x^{*}, y^{*}, z^{*}\right)=(0,1,0)$.

(II) the case that $x=0$ :

(a) $2 y \xi_{1}-\xi_{2}>0:\left(x^{*}, y^{\prime \prime}, z^{*}\right):\left\{(1-\alpha, \alpha, 0) \mid \alpha \leqq \frac{\xi_{2}}{2 y \xi_{1}}, y>0, \xi_{1}>0, \xi_{2}>0, \xi_{1}+\xi_{2}=1\right\}$.

(b) $2 y \xi_{1}-\xi_{2}=0:\left(x^{*}, y^{*}, z^{*}\right):\{(\alpha, 1-\alpha, 0) \mid 0 \leqq \alpha \leqq 1\}$.

(c) $2 y \xi_{1}-\xi_{2}<0:\left(x^{*}, y^{*}, z^{*}\right):\{(\alpha, 1-\alpha, 0) \mid 0 \leqq \alpha \leqq 1\}$. 
(III) the case that $x<0$ :

(a) $2 y \xi_{1}-\xi_{2}>0$ :

$$
\begin{aligned}
&\left(x^{\prime \prime}, y^{\prime \prime}, z^{*}\right):\left\{\left(\frac{2 y \xi_{1}-\xi_{2}}{2 y \xi_{1}-2 x \xi_{1}-2 x y \xi_{1}+x \xi_{2}},-\frac{2 x \xi_{1}-\xi_{2}}{2 y \xi_{1}-2 x \xi_{1}-2 x y \xi_{1}+x \xi_{2}},-\frac{2 x y \xi_{1}-x \xi_{2}}{2 y \xi_{1}-2 x \xi_{1}-2 x y \xi_{1}+x \xi_{2}}\right):\right. \\
&\left.2 y \xi_{1}-2 x \xi_{1}-2 x y \xi_{1}+x \xi_{2}>0,2 y \xi_{1}-\xi_{2}>0,2 x \xi_{1}-\xi_{2}<0, \xi_{1}>0, \xi_{2}>0, \xi_{1}+\xi_{2}=1\right\} . \\
& \text { (b) } 2 y \xi_{1}-\xi_{2}=0:\left(x^{*}, y^{*}, z^{*}\right):\{(\alpha, 1-\alpha, 0) \mid 0 \leqq \alpha \leqq 1\} . \\
& \text { (c) } 2 y \xi_{1}-\xi_{2}<0:\left(x^{*}, y^{*}, z^{*}\right)=(1,0,0) .
\end{aligned}
$$

Let $(x, y) \in \mathbb{R}^{2}$ and $S_{(x, y)}$ be the set of vector solutions of the vector matrix game $B_{i}(x, y)$, $i=1,2$. From (I), (II) and (III),

$$
\begin{aligned}
\bigcup_{(x, y) \in \mathbb{R}^{2}} S(x, y)= & \{(\alpha, 1-\alpha, 0) \mid 0 \leqq \alpha \leqq 1\} \cup\{(0, \alpha, 1-\alpha) \mid 0 \leqq \alpha \leqq 1\} \\
& \cup\{(\alpha, 0,1-\alpha) \mid 0 \leqq \alpha \leqq 1\} \\
& \cup\{(\alpha, \beta, \gamma) \mid \alpha \geqq 0, \beta \geqq 0, \gamma \geqq 0, \alpha+\beta+\gamma=1\} \\
& \cup\left\{\left(\frac{2 y \xi_{1}-\xi_{2}}{2 y \xi_{1}-2 x \xi_{1}-2 x y \xi_{1}+x \xi_{2}},-\frac{2 x \xi_{1}-\xi_{2}}{2 y \xi_{1}-2 x \xi_{1}-2 x y \xi_{1}+x \xi_{2}},\right.\right. \\
& \left.-\frac{2 x y \xi_{1}-x \xi_{2}}{2 y \xi_{1}-2 x \xi_{1}-2 x y \xi_{1}+x \xi_{2}}\right) \mid x<0,2 y \xi_{1}-2 x \xi_{1}-2 x y \xi_{1}+x \xi_{2}>0, \\
& \left.2 y \xi_{1}-\xi_{2}>0,2 x \xi_{1}-\xi_{2}<0, \xi_{1}>0, \xi_{2}>0, \xi_{1}+\xi_{2}=1\right\} .
\end{aligned}
$$

Let $(\bar{x}, \bar{y}, \bar{\xi})$ be feasible for (SP) and (SD) with $\bar{y} \nabla_{y}\left(\bar{\xi}^{T} f\right)(\bar{x}, \bar{y})=\bar{x} \nabla_{x}\left(\bar{\xi}^{T} f\right)(\bar{x}, \bar{y})=0$. We can easily check that

$$
\begin{aligned}
& \left\{(x, y, \xi) \mid(x, y, \xi) \text { is feasible for (SP) and (SD), } \bar{y} \nabla_{y}\left(\xi^{T} f\right)(x, y)=\bar{x} \nabla_{x}\left(\xi^{T} f\right)(x, y)=0\right\} \\
& \quad=\left\{\left(\frac{\xi_{2}}{2 \xi_{1}}, \frac{\xi_{2}}{2 \xi_{1}}, \xi_{1}, \xi_{2}\right) \mid \xi_{1}>0, \xi_{2}>0, \xi_{1}+\xi_{2}=1\right\} .
\end{aligned}
$$

Thus,

$$
\begin{aligned}
& \left(\frac{\bar{x}}{1+\bar{x}+\bar{y}}, \frac{\bar{y}}{1+\bar{x}+\bar{y}}, \frac{1}{1+\bar{x}+\bar{y}}\right) \\
& \quad \in\left\{\left(\frac{\xi_{2}}{2}, \frac{\xi_{2}}{2}, \xi_{1}\right) \mid \xi_{1}>0, \xi_{2}>0, \xi_{1}+\xi_{2}=1\right\} \\
& \quad \subset S_{(\bar{x}, \bar{y}) .}
\end{aligned}
$$

Therefore, Theorem 3.1 holds.

Let $(x, y) \in \mathbb{R}^{2}$ and $S_{(x, y)}$ be the set of vector solutions of the vector matrix game $B_{i}(x, y)$, $i=1,2$. Then

$$
\begin{aligned}
\bigcup_{(x, y) \in \mathbb{R}^{2}} S_{(x, y)}= & \{(\alpha, 1-\alpha, 0) \mid 0 \leqq \alpha \leqq 1\} \cup\{(0, \alpha, 1-\alpha) \mid 0 \leqq \alpha \leqq 1\} \\
& \cup\{(\alpha, 0,1-\alpha) \mid 0 \leqq \alpha \leqq 1\} \\
& \cup\{(\alpha, \beta, \gamma) \mid \alpha \geqq 0, \beta \geqq 0, \gamma \geqq 0, \alpha+\beta+\gamma=1\} \\
& \cup\left\{\left(\frac{2 y \xi_{1}-\xi_{2}}{2 y \xi_{1}-2 x \xi_{1}-2 x y \xi_{1}+x \xi_{2}},-\frac{2 x \xi_{1}-\xi_{2}}{2 y \xi_{1}-2 x \xi_{1}-2 x y \xi_{1}+x \xi_{2}},\right.\right.
\end{aligned}
$$




$$
\begin{aligned}
& \left.-\frac{2 x y \xi_{1}-x \xi_{2}}{2 y \xi_{1}-2 x \xi_{1}-2 x y \xi_{1}+x \xi_{2}}\right) \mid x<0,2 y \xi_{1}-2 x \xi_{1}-2 x y \xi_{1}+x \xi_{2}>0 \\
& \left.2 y \xi_{1}-\xi_{2}>0,2 x \xi_{1}-\xi_{2}<0, \xi_{1}>0, \xi_{2}>0, \xi_{1}+\xi_{2}=1\right\}
\end{aligned}
$$

So,

$$
\left\{\left(\frac{x^{*}}{z^{*}}, \frac{y^{*}}{z^{*}}\right) \mid z^{*}>0 \text { and }\left(x^{*}, y^{*}, z^{*}\right) \in S_{\left(\frac{x^{*}}{z^{*}}, \frac{y^{*}}{z^{*}}\right)}\right\}=\left\{\left(\frac{\xi_{2}}{2 \xi_{1}}, \frac{\xi_{2}}{2 \xi_{1}}\right) \mid \xi_{1}>0, \xi_{2}>0, \xi_{1}+\xi_{2}=1\right\} \text {. }
$$

Let $F$ be the set of all feasible solutions of (SP) and let $G$ be the set of all feasible solutions of (SD). Then we can check that $\left\{\left(\frac{\xi_{2}}{2 \xi_{1}}, \frac{\xi_{2}}{2 \xi_{1}}, \xi_{1}, \xi_{2}\right) \mid \xi_{1}>0, \xi_{2}>0, \xi_{1}+\xi_{2}=1\right\} \subset F \cap G$ and $\left(\frac{\xi_{2}}{2 \xi_{1}}\right) \nabla_{y}\left(\xi^{T} f\right)\left(\frac{\xi_{2}}{2 \xi_{1}}, \frac{\xi_{2}}{2 \xi_{1}}\right)=\left(\frac{\xi_{2}}{2 \xi_{1}}\right) \nabla_{x}\left(\xi^{T} f\right)\left(\frac{\xi_{2}}{2 \xi_{1}}, \frac{\xi_{2}}{2 \xi_{1}}\right)=0$. Therefore, Theorem 3.2 holds.

\section{Competing interests}

The authors declare that they have no competing interests.

\section{Authors' contributions}

The authors, together discussed and solved the problems in the manuscript. All authors read and approved the final manuscript.

\section{Author details}

'Department of Applied Mathematics, Pukyong National University, Busan, 608-737, Korea. ${ }^{2}$ School of Free Major, Tongmyong University, Busan, 608-711, Korea.

\section{Acknowledgements}

The authors would like to thank the referees for giving valuable comments for the revision of the paper.

Received: 30 June 2012 Accepted: 26 November 2012 Published: 28 December 2012

\section{References}

1. Dantzig, GB: A proof of the equivalence of the programming problem and the game problem. In: Koopmans, TC (ed.) Activity Analysis of Production and Allocation. Cowles Commission Monograph, vol. 13, pp. 330-335. Wiley, New York (1951)

2. Chandra, S, Craven, BD, Mond, B: Nonlinear programming duality and matrix game equivalence. J. Aust. Math. Soc. Ser. B, Appl. Math 26, 422-429 (1985)

3. Chandra, S, Mond, B, Duraga Prasad, MV: Continuous linear programs and continuous matrix game equivalence. In: Kumar, S (ed.) Recent Developments in Mathematical Programming, pp. 397-406. Gordan and Breach Science Publishers, New York (1991)

4. Kim, DS, Noh, K: Symmetric dual nonlinear programming and matrix game equivalence. J. Math. Anal. Appl. 298, 1-13 (2004)

5. Preda, V: On nonlinear programming and matrix game equivalence. J. Aust. Math. Soc. Ser. B, Appl. Math 35, 429-438 (1994)

6. Hong, JM, Kim, MH: On vector optimization problem and vector matrix game equivalence. J. Nonlinear Convex Anal. 12(3), 651-662 (2011)

7. Dorn, WS: A symmetric dual theorem for quadratic programs. J. Oper. Res. Soc. Jpn. 2, 93-97 (1960)

8. Dantzig, GB, Eisenberg, E, Cottle, RW: Symmetric dual nonlinear programs. Pac. J. Math. 15, 809-812 (1965)

9. Mond, B, Weir, T: Symmetric duality for nonlinear multiobjective programming. Q. J. Mech. Appl. Math. 23, 265-269 (1965)

10. Sawaragi, Y, Nakayama, H, Tanino, T: Theory of Multiobjective Optimization. Academic Press, Orlando (1985)

11. Aubin, JP: Mathematical Methods of Game and Economic Theory. North-Holland, Amsterdam (1979)

doi:10.1186/1687-1812-2012-233

Cite this article as: Hong et al.: On vector matrix game and symmetric dual vector optimization problem. Fixed Point Theory and Applications 2012 2012:233. 